Bull. Korean Math. Soc. 49 (2012), No. 1, pp. 49-55

http://dx.doi.org/10.4134/BKMS.2012.49.1.049

\title{
CHARACTERIZING COMPLEX LOCALLY MINKOWSKI SPACES BY HOLOMORPHIC SECTIONAL CURVATURE
}

\author{
Xinxiang Chen and Rongmu Yan
}

\begin{abstract}
In this paper, we prove that a complex Finsler manifold is a complex locally Minkowski space if and only if it is a strictly KählerBerwald manifold with zero holomorphic sectional curvature.
\end{abstract}

\section{Introduction}

Recently, more and more people have been attracted to the study of Finsler geometry. The study of Finsler spaces has many applications in physics and biology. In complex Finsler geometry, people think the notion of Kähler-Finsler metrics is the extension of the Kähler metrics. Actually, the Kähler-Berwald metrics may be the closest non-Hermitian complex Finsler metrics to the Kähler metrics. Therefore, to explore the properties of the Kähler-Finsler metrics and the Kähler-Berwald metrics is one of the most important tasks in complex Finsler geometry.

Here we will describe the characterization of the Kähler-Berwald manifolds with zero holomorphic sectional curvature.

Main Theorem. Let $F$ be a complex Finsler metric on a complex manifold $M$. Then it is a complex locally Minkowski metric if and only if it is a strictly Kähler-Berwald metric with vanishing holomorphic sectional curvature.

A complex Finsler metric is a strictly Kähler-Finsler metric in the sense that it is a Kähler-Finsler metric satisfying the following condition on the torsion of the induced Chern-Finsler connection by $F$ :

$$
\left\langle\bar{\partial}_{H} \theta(H, \chi, \bar{K}), \chi\right\rangle=0 \quad \text { for all } H, K \in \mathcal{H},
$$

where $\mathcal{H}$ refer to the $(1,0)$-part of complex horizontal bundle. M. Abate and G. Patrizio [1] have ever used this condition to discuss those complex Finsler

Received January 1, 2010.

2010 Mathematics Subject Classification. 53C56, 32Q99.

Key words and phrases. complex Berwald manifold, holomorphic sectional curvature, complex locally Minkowski space.

Supported by the Fundamental Research Funds for the Central Universities (2010121002, 2011121040). 
metrics with nonpositive constant holomorphic sectional curvature. Notice that it is different from the definition of a strongly Kähler-Finsler metric.

A special case of the main theorem has been proved by the second author $[13,14]$ with a stronger assumption.

Here we refer to [1] and [13] for all other definitions and notations.

\section{Proof of the main theorem}

From now on, we assume $F$ is a strictly Kähler-Berwald metric on a complex manifold $M$ with zero holomorphic sectional curvature. Using the coefficients $\Gamma_{\beta ; \gamma}^{\alpha}$, we define the complex Berwald connection:

$$
D V=\left(\frac{\partial V^{\alpha}}{\partial z^{\gamma}}+V^{\beta} \Gamma_{\beta ; \gamma}^{\alpha}\right) \frac{\partial}{\partial z^{\alpha}} \otimes d z^{\gamma}
$$

for a holomorphic vector field $V=V^{\alpha} \frac{\partial}{\partial z^{\alpha}}$. Obviously, $D$ is a linear connection since $F$ is complex Berwaldian.

The curvature forms of $D$ are

$$
\Omega_{\alpha}^{\beta}=d \omega_{\alpha}^{\beta}-\omega_{\alpha}^{\gamma} \wedge \omega_{\gamma}^{\beta},
$$

where $\omega_{\alpha}^{\beta}=\Gamma_{\gamma ; \alpha}^{\beta} d z^{\gamma}$.

Under local coordinate system, we can write

$$
\Omega_{\alpha}^{\beta}=\frac{1}{2} K_{\alpha \gamma \delta}^{\beta} d z^{\gamma} \wedge d \bar{z}^{\delta}
$$

where $K_{\alpha \gamma \bar{\delta}}^{\beta}=-2 \frac{\partial \Gamma_{\alpha ; \gamma}^{\beta}}{\partial \bar{z}^{\delta}}$, since $\left[\frac{\delta}{\delta z^{\mu}}, \frac{\delta}{\delta z^{\nu}}\right]=0$.

We know from $[1,3,12]$ that the holomorphic sectional curvature of $(M, F)$ is

$$
K(X)=-\frac{G_{\alpha \bar{\beta}} K_{\sigma \gamma \bar{\delta}}^{\alpha} y^{\sigma} \bar{y}^{\beta} y^{\gamma} \bar{y}^{\delta}}{2 G^{2}(y)},
$$

where $X \in T_{p} M, p \in M$, and $X=y+\bar{y}, y \in T_{p}^{1,0} M, y=y^{\alpha} \frac{\partial}{\partial z^{\alpha}}$. It is clear that the holomorphic sectional curvature of $D$ is equal to that of $F$.

Since $(M, F)$ is a Kähler-Berwald manifold, $\Gamma_{\beta ; \mu}^{\alpha}$, and $K_{\alpha \gamma \bar{\delta}}^{\beta}$ is independent on $y$.

Let $\nabla$ be the Chern-Finsler connection associated to $F$. In local coordinates, the curvature operator of $\nabla$ is given by

$$
\Omega_{\beta}^{\alpha}=R_{\beta ; \mu \bar{\nu}}^{\alpha} d z^{\mu} \wedge d \bar{z}^{\nu}+R_{\beta \delta ; \bar{\nu}}^{\alpha} \psi^{\delta} \wedge d \bar{z}^{\nu}+R_{\beta \bar{\gamma} ; \mu}^{\alpha} d z^{\mu} \wedge \bar{\psi}^{\gamma}+R_{\beta \delta \bar{\gamma}}^{\alpha} \psi^{\delta} \wedge \bar{\psi}^{\gamma}
$$

where

$$
\begin{aligned}
R_{\beta ; \mu \bar{\nu}}^{\alpha} & =-\delta_{\bar{\nu}}\left(\Gamma_{\beta ; \mu}^{\alpha}\right)-\Gamma_{\beta \sigma}^{\alpha} \delta_{\bar{\nu}}\left(\Gamma_{; \mu}^{\sigma}\right), \\
R_{\beta \delta ; \bar{\nu}}^{\alpha} & =-\delta_{\bar{\nu}}\left(\Gamma_{\beta \delta}^{\alpha}\right), \\
R_{\beta ; \bar{\gamma} \mu}^{\alpha} & =-\dot{\partial}_{\gamma}\left(\Gamma_{\beta ; \mu}^{\alpha}\right)-\Gamma_{\beta \sigma}^{\alpha} \Gamma_{\bar{\gamma} ; \mu}^{\sigma}, \\
R_{\beta \delta \bar{\gamma}}^{\alpha} & =-\dot{\partial}_{\gamma}\left(\Gamma_{\beta \delta}^{\alpha}\right) .
\end{aligned}
$$

The notations here are similar to [1]. 
Since $(M, F)$ is with zero holomorphic sectional curvature, then

$$
G_{\alpha \bar{\beta}} K_{\sigma \gamma \bar{\delta}}^{\alpha} y^{\sigma} \bar{y}^{\beta} y^{\gamma} \bar{y}^{\delta}=0 .
$$

Denote $K_{\beta \bar{\sigma} \mu \bar{\nu}}=G_{\alpha \bar{\sigma}} K_{\beta \mu \bar{\nu}}^{\alpha}, R_{\beta \bar{\sigma} ; \mu \bar{\nu}}=G_{\alpha \bar{\sigma}} R_{\beta ; \mu \bar{\nu}}^{\alpha}$, then

$$
R_{\beta \bar{\sigma} ; \mu \bar{\nu}}=\frac{1}{2} K_{\beta \bar{\sigma} \mu \bar{\nu}}-G_{\alpha \bar{\sigma}} \Gamma_{\beta \delta}^{\alpha} \delta_{\bar{\nu}}\left(\Gamma_{; \mu}^{\delta}\right)
$$

and

$$
R_{\beta \bar{\sigma} ; \mu \bar{\nu}} y^{\beta}=\frac{1}{2} K_{\beta \bar{\sigma} \mu \bar{\nu}} y^{\beta} .
$$

By Lemma 3.1.6 in [1], condition (1) is equivalent to

$$
\langle\Omega(H, \bar{K}) \chi, \chi\rangle=\langle\Omega(\chi, \bar{K}) H, \chi\rangle \quad \text { for all } H, K \in \mathcal{H} .
$$

Under local coordinates, it is

$$
R_{\beta \bar{\sigma} ; \mu \bar{\nu}} y^{\beta} \bar{y}^{\nu}=R_{\mu \bar{\sigma} ; \beta \bar{\nu}} y^{\beta} \bar{y}^{\nu} .
$$

Furthermore, by (1.4) in [3],

$$
\overline{R_{\beta \bar{\sigma} ; \mu \bar{\nu}}}=R_{\sigma \bar{\beta} ; \nu \bar{\mu}} .
$$

Notice that $K_{\beta \bar{\sigma} \mu \bar{\nu}}=K_{\mu \bar{\sigma} \beta \bar{\nu}}$, so we have

$$
K_{\beta \bar{\sigma} \mu \bar{\nu}} y^{\beta} y^{\mu} \bar{y}^{\nu}=K_{\beta \bar{\nu} \mu \bar{\sigma}} y^{\beta} y^{\mu} \bar{y}^{\nu} .
$$

Differentiating on $\bar{y}$ for both sides of (3), it turns into

$$
K_{\sigma \bar{\beta} \gamma \bar{\delta}} y^{\sigma} y^{\gamma} \bar{y}^{\delta}=0,
$$

where we have used (5). It turns into

$$
K_{\sigma \gamma \delta}^{\alpha} y^{\sigma} y^{\gamma} \bar{y}^{\delta}=0
$$

Differentiating again on $\bar{y}$ and $y$, we can have

$$
K_{\sigma \gamma \bar{\delta}}^{\alpha}=0
$$

For any fixed point $p \in M$, let $\left(z^{i}\right)$ be any local holomorphic coordinate system on some open set $U$ with $z^{i}(p)=0$. For any holomorphic vector $X(p) \in$ $T_{p}^{1,0} M$, we want to extend it to a covariantly constant holomorphic vector field $X$ (that is, $D X=0$ ) on $U$. Let $\gamma\left(z^{1}\right):=\left(z^{1}, 0, \ldots, 0\right)$ be the $z^{1}$-coordinate complex surface that passes through $p, z^{1}=x^{1}+i y^{1}$. Since $D$ is linear, we can parallel translate $X(p)$ along the real axis. At each point along the real axis, we now have a holomorphic vector. Parallel translate this vector along the direction parallel to the imaginary axis, we have thus extended $X(p)$ to a holomorphic vector field $X\left(z^{1}, 0, \ldots, 0\right)$ on the $z^{1}$-coordinate complex surface $\Sigma_{1}:=\left\{z \mid z=\left(z^{1}, 0,0, \ldots, 0\right) \in U\right\}$ that passes through $p$.

Since the holomorphic sectional curvature of $D$ vanishes, we have

$$
D_{\frac{\partial}{\partial x^{1}}} D_{\frac{\partial}{\partial y^{1}}} X-D_{\frac{\partial}{\partial y^{1}}} D_{\frac{\partial}{\partial x^{1}}} X=0 \quad \text { on } \Sigma_{1} \text {. }
$$


But by construction, $D_{\frac{\partial}{\partial y^{1}}} X=0$ on $\Sigma_{1}$. So $D_{\frac{\partial}{\partial x^{1}}} X$ is parallel along the imaginary coordinate curve, and it vanishes at the points on the real coordinate curve. Thus it must be identically zero along the $z^{1}$-coordinate complex surface. Hence $D_{\frac{\partial}{\partial z^{1}}} X=0$ on $\Sigma_{1}$.

By following the similar step as above, we can extend $X$ onto $\Sigma_{2}:=\{z \mid z=$ $\left.\left(z^{1}, z^{2}, 0, \ldots, 0\right) \in U\right\}$. Obviously,

$$
D_{\frac{\partial}{\partial z^{2}}} X=0 \quad \text { on } \quad \Sigma_{2} \text {. }
$$

We would show that $D_{\frac{\partial}{\partial z^{1}}} X=0$ is also true on $\Sigma_{2}$.

We digress to construct a linear connection on $M$ as a real manifold. Let $\left\{z^{1}, \ldots, z^{n}\right\}$ be a set of local coordinates, with $z^{\alpha}=x^{\alpha}+i x^{n+\alpha}$, so that $\left\{x^{1}, \ldots, x^{n}, x^{n+1}, \ldots, x^{2 n}\right\}$ are local real coordinates. We use the same convention as [13]: Lowercase greek indices will run from 1 to $n$, whereas lowercase roman indices will run from 1 to $2 n$.

We write $\omega_{\alpha}^{\beta}=\theta_{\alpha}^{\beta}+\sqrt{-1} \theta_{\alpha}^{n+\beta}$, and let $\theta_{n+\alpha}^{n+\beta}=\theta_{\alpha}^{\beta}, \theta_{n+\alpha}^{\beta}=-\theta_{\alpha}^{n+\beta}$. These $\left\{\theta_{a}^{b}\right\}$ can be used as a connection form on $M$, i.e., we define

$$
\hat{D} \frac{\partial}{\partial x^{a}}=\theta_{a}^{b} \frac{\partial}{\partial x^{b}} .
$$

If we write $\theta_{a}^{b}=\hat{\Gamma}_{c a}^{b} d x^{c}$, the relation of $\Gamma_{\gamma ; \alpha}^{\beta}$ and $\hat{\Gamma}_{c a}^{b}$ is just the (5) and (6) in [13]. It is easily known that $D$ can be looked as the complexified linear extension of $\hat{D}$.

The curvature form of $\hat{D}$ is

$$
\Theta_{a}^{b}=d \theta_{a}^{b}-\theta_{a}^{c} \wedge \theta_{c}^{b} .
$$

Obviously,

$$
\Omega_{\alpha}^{\beta}=\Theta_{\alpha}^{\beta}+\sqrt{-1} \Theta_{\alpha}^{n+\beta}
$$

and

$$
\Theta_{n+\alpha}^{n+\beta}=\Theta_{\alpha}^{\beta} \quad \Theta_{n+\alpha}^{\beta}=-\Theta_{\alpha}^{n+\beta} .
$$

If we denote the curvature tensors of $D$ and $\hat{D}$ by $R$ and $\hat{R}$ respectively, then $R$ is also the complexified linear extension of $\hat{R}$.

Now we return to our proof.

Since $D$ is with vanishing holomorphic sectional curvature, then $\Omega_{\alpha}^{\beta}=0$ and $\Theta_{a}^{b}=0$ by the above discussion. Hence both the curvature tensor $\hat{R}$ and $R$ vanish. Now we have

$$
D_{\frac{\partial}{\partial z^{1}}} D_{\frac{\partial}{\partial z^{2}}} X-D_{\frac{\partial}{\partial z^{2}}} D_{\frac{\partial}{\partial z^{1}}} X=0 \quad \text { on } \quad \Sigma_{2} .
$$

By this formula and (6), $D_{\frac{\partial}{\partial z^{1}}} X$ is parallel along the $z^{2}$-coordinate and it vanishes at the points on the $\Sigma_{1}:=\left\{\left(z^{1}, 0, \ldots, 0\right) \in U\right\}$. Hence $D_{\frac{\partial}{\partial z^{1}}} X=0$ on $\Sigma_{2}$. 
This process of extension can be continued until one obtains a holomorphic vector field $X$ on $U$ satisfying $D_{\frac{\partial}{\partial z^{\alpha}}} X=0$ for all $\alpha$. And this last property is equivalent to $D_{Y} X=0$ for all holomorphic vector fields $Y$.

Now we take any basis $\left\{X_{\alpha}(p)\right\}$ of $T_{p}^{1,0} M$ and, through the above procedure, extend it to a collection of covariantly constant holomorphic vector fields $\left\{X_{\alpha}\right\}$ on $U$. These holomorphic vector fields form a basis at every holomorphic tangent space in $U$. Also, since a Kähler-Finsler metric must be strongly Kähler-Finslerian for a complex Berwald metric and the torsion must vanish for a strongly Kähler-Finsler metric, it implies that $\left[X_{\alpha}, X_{\beta}\right]=0$. So one can construct local coordinates $\left(z^{\alpha}\right)$ on $U$ such that $X_{\alpha}=\frac{\partial}{\partial z^{\alpha}}$. The statement $D_{X_{\gamma}} X_{\alpha}=0$ then implies that $\Gamma_{\gamma ; \alpha}^{\beta}=0$. So under this coordinate, it gives $\frac{\partial G_{\alpha \bar{\beta}}}{\partial z^{\gamma}}=0$. And $(M, F)$ is a complex locally Minkowski space.

Conversely, suppose $(M, F)$ is a complex locally Minkowski space. Obviously $\Gamma_{; \alpha}^{\beta}$ vanishes in some privileged coordinate charts and so $\Gamma_{\gamma ; \alpha}^{\beta}$. This means $(M, F)$ is a strictly Kähler-Berwald space with vanishing holomorphic sectional curvature. And we finish our proof of the main theorem.

\section{Two examples of complex locally Minkowski spaces}

Example 1. Let $M$ be a complex manifold with an Hermitian metric $\alpha$, and $\beta$ be a $(1,0)$-form on $M$. If a function $F(\alpha, \beta)$ satisfies the conditions in Definition 2.3.1 and Definition 2.3.5 in [1] as a function from $T^{1,0} M$ to $\mathbb{R}^{+}$, then $F(\alpha, \beta)$ is called a complex $(\alpha, \beta)$-metric on $M$.

We know from [2] that if the $(1,0)$-form $\beta$ is holomorphic and parallel with respect to the Hermite connection induced by $\alpha$, then $(M, F(\alpha, \beta))$ is a complex Berwald manifold. If we further assume $\alpha$ is a Kähler metric, then $(M, F(\alpha, \beta))$ is a Kähler-Finsler manifold. Under these conditions, if $\alpha$ is also with vanishing holomorphic sectional curvature, then $\Gamma_{\beta ; \gamma}^{\alpha}=0$. Hence $(M, F(\alpha, \beta)$ is a strictly Kähler-Berwald manifold with zero holomorphic sectional curvature, and it is a complex locally Minkowski space by the main theorem.

Example 2. Let $\left(M_{1}, \alpha\right),\left(M_{2}, \beta\right)$ be Hermitian manifolds. $F_{\varepsilon}(\varepsilon>0)$ is the complex Szabó metric on the product manifold $M_{1} \times M_{2}$ defined by

$$
F_{\varepsilon}:=\sqrt{\alpha\left(y_{1}\right)^{2}+\beta\left(y_{2}\right)^{2}+\varepsilon\left(\alpha\left(y_{1}\right)^{2 k}+\beta\left(y_{2}\right)^{2 k}\right)^{\frac{1}{k}}},
$$

where $y=y_{1} \oplus y_{2}=\left(v^{1}, \ldots, v^{m}, v^{m+1}, \ldots, v^{m+n}\right) \in T_{z}^{1,0}\left(M_{1} \times M_{2}\right), z=$ $\left(z_{1}, z_{2}\right) \in M_{1} \times M_{2}, y_{1}=\left(v^{1}, \ldots, v^{m}\right) \in T_{z_{1}}^{1,0} M_{1}, y_{2}=\left(v^{m+1}, \ldots, v^{m+n}\right) \in$ $T_{z_{2}}^{1,0} M_{2}$, and $k>1$ is a positive integer.

We have known in [5] that $F_{\varepsilon}$ is a strongly pseudoconvex complex Finsler metric. Furthermore, $F_{\varepsilon}$ is strongly Kähler-Finslerian if $\alpha$ and $\beta$ are both Kähler metrics. In fact, the coefficients of Chern-Finsler connection can be 
written as follows:

$$
N_{j}^{i}(y)= \begin{cases}\sum_{l=1}^{m} a^{\bar{l} i} v_{\bar{l} ; j} & 1 \leq i, j \leq m \\ \sum_{l=m+1}^{m+n} b^{l i} v_{\bar{l} ; j} & m+1 \leq i, j \leq m+n \\ 0 & \text { otherwise }\end{cases}
$$

For $X=\left(X_{1}, X_{2}\right)=y+\bar{y} \in T_{z}\left(M_{1} \times M_{2}\right)$, by a direct computation, we have

$$
\begin{aligned}
& K(z, X) \\
= & \frac{1}{G^{2}}\left(A a_{\alpha \bar{\delta}} \Gamma_{\gamma ; \mu \bar{\nu}}^{\alpha} v^{\gamma} \bar{v}^{\delta} v^{\mu} \bar{v}^{\nu}+B b_{\alpha+m \overline{\delta+m}} \Gamma_{\gamma+m ; \mu+m \overline{\nu+m}}^{\alpha+m} v^{\gamma+m} \bar{v}^{\delta+m}\right) v^{\mu+m} \bar{v}^{\nu+m} \\
= & \frac{1}{G^{2}}\left(A K_{\alpha}\left(z_{1}, X_{1}\right)+B K_{\beta}\left(z_{2}, X_{2}\right)\right)
\end{aligned}
$$

where $\left.G=F_{\varepsilon}^{2}, A=1+\varepsilon\left(\alpha^{2 k}+\beta^{2 k}\right)\right)^{\frac{1}{k}-1} \alpha^{2(k-1)}, B=1+\varepsilon\left(\alpha^{2 k}+\beta^{2 k}\right)^{\frac{1}{k}-1} \beta^{2(k-1)}$.

Now we can easily know that $\left(M_{1} \times M_{2}, F_{\varepsilon}\right)$ is a complex locally Minkowski space if both $\left(M_{1}, \alpha\right)$ and $\left(M_{2}, \beta\right)$ have vanishing holomorphic sectional curvatures.

\section{References}

[1] M. Abate and G. Patrizio, Finsler Metrics-A Global Approach, with applications to geometric function theory, Lecture Notes in Mathematics, 1591. Springer-Verlag, Berlin, 1994.

[2] T. Aikou, On complex Finsler manifolds, Rep. Fac. Sci. Kagoshima Univ. Math. Phys. Chem. (1991), no. 24, 9-25.

[3] N. Aldea, The holomorphic bisectional curvature of the complex Finsler spaces, Novi Sad J. Math. 35 (2005), no. 2, 143-153.

[4] D. Bao, S. S. Chern, and Z. Shen, An Introduction to Riemann-Finsler Geometry, GTM 200, Springer-Verlag, New York, 2000.

[5] Y. Chen and R. Yan, The Szabó metric on product of complex manifolds, Acta Math. Sinica (Chin. Ser.) 50 (2007), no. 4, 801-804.

[6] S. S. Chern and Z. Shen, Riemann-Finsler Geometry, World scientific, Singapore, 2005.

[7] Z. Shen, Volume comparison and its applications in Riemann-Finsler geometry, Adv. Math. 128 (1997), no. 2, 306-328.

[8] Z. Szabó, Positive definite Berwald spaces. Structure theorems on Berwald spaces, Tensor (N.S.) 35 (1981), no. 1, 25-39.

[9] J. Xiao and R. Yan, Two topics in complex Finsler geometry, Xiamen Daxue Xuebao Ziran Kexue Ban 45 (2006), no. 5, 614-616.

[10] R. Yan, Connections on complex Finsler manifold, Acta Math. Appl. Sin. Engl. Ser. 19 (2003), no. 3, 431-436.

[11] $\ldots$, On the volume of the projectivized tangent bundle in a complex Finsler manifold, Arch. Math. (Basel) 86 (2006), no. 5, 458-463.

[12] - Holomorphic sectional curvature in complex Finsler geometry, Preprint.

[13] _ Complex Berwald manifolds with vanishing holomorphic sectional curvature, Glasg. Math. J. 50 (2008), no. 2, 203-208.

[14] _ Complex Berwald manifolds with vanishing holomorphic sectional curvaturecorrigendum, Glasg. Math. J. 52 (2010), no. 1, 205. 


\section{XiNXIANG CHEN}

School of Mathematical Science

Xiamen University

Fujian Province, 361005, P. R. China

E-mail address: xxchen@xmu.edu.cn

RONGMU YAN

School of Mathematical Science

Xiamen University

Fujian Province, 361005, P. R. China

E-mail address: yanrm@xmu.edu.cn 